\section{Heschl's Gyrus}

John E. Mendoza

Department of Psychiatry and Neuroscience, Tulane Medical School and SE Louisiana

Veterans Healthcare System, New Orleans, LA, USA

\section{Synonyms}

Primary auditory cortex; Transverse gyri of Heschl

\section{Definition}

That portion of the superior temporal gyrus that lies inside the banks of the lateral fissure (the temporal operculum). This area is also referred to as the transverse gyri of Heschl as the individual gyri are oriented in a lateral-medial plane within the operculum (as opposed to a more anterior-posterior or inferior-superior plane of most of the cortical gyri). This region is the primary cortical projection area for auditory information coming from the medial geniculates, the specific auditory relay nuclei of the thalamus. Because of the extensive bilateral projections that characterize the auditory system, unilateral lesions restricted to Heschl's gyrus would be expected to produce only very subtle deficits and may be limited to increased difficulty with sound localization. While quite rare, bilateral lesions to this structure could result in deafness.

\section{Cross-References}

Auditory System 